\title{
Rescate con Sirolimus por rechazo agudo recurrente y diabetes mellitus en trasplante hepático. Caso clínico pediátrico
}

\author{
Jorge Roque E, G loria Ríos M, Juan Hepp K, \\ Roberto Humeres A, Horacio Ríos R, José M Herrera, \\ Montserrat Rius $A^{a}$. \\ Sirolimus for rescue of recurrent \\ acute rejection and diabetes mellitus \\ after liver transplantation. \\ Report of one case
}

Sirolimus (SRL) is a new immunosuppressive drug approved for renal transplantation, but is being used increasingly in orthotopic liver transplantation (OLT). Compared with the calcineurin inhibitors, SRL has different mechanisms of action and side effects profile. Thus, this drug offers significant potential advantages over other immunosuppressive agents. SRL does not cause glucose intolerance, hypertension or renal failure, but it may cause dyslipidemia, hepatic artery thrombosis, thrombocytopenia, anemia, leukopenia, oral mucosa ulcers, edema, arthralgias and wound complications. SRL inhibits the signal of interleukin 2 at a post-receptor level, inhibiting lymphocyte proliferation and fibroblast proliferation. It also has antineoplastic and antifungal effects. We report a 10 years old girl who underwent OLT, experiencing a biopsy-proven recurrent acute rejection (AR) in spite of using three immunosuppressive agents (tacrolimus, mofetil micofenolate and steroids). She developed diabetes mellitus as a consequence of the immunosuppressive therapy. She was rescued with SRL, not experiencing AR again. Mofetil micofenolate, steroids and insulin could be discontinued and tacrolimus doses were reduced, without experiencing severe complications. SRL is a new and safe immunosuppressive agent for rescue in patients with OLT and recurrent AR (Rev Méd Chile 2005; 133: 1221-4).

(Key Words: Liver transplantation; Sirolimus; Tacrolimus)

Recibido el 20 de marzo, 2005. Aceptado el 30 de junio, 2005. Unidad de Trasplante, Clínica Alemana de Santiago de Chile. aEnfermera Universitaria

Correspondencia a: Dr. Jorge Roque E. Avenida

Manquehue 1410, piso 1, Vitacura. Fax: 2124380.

E-mail: j.roque@entelchile.net 
L a inmunosupresión en los niños tiene una serie de efectos secundarios, entre los que se cuentan diabetes mellitus (DM), enfermedad linfoproliferativa post trasplante (ELPT), neurotoxicidad y nefrotoxicidad en el caso de los inhibidores de los calcineunínicos (IC) ${ }^{1,2}$ o retardo del crecimiento con los esteroides ${ }^{3}$. Sirolimus (SRL) que fue inicialmente aprobado para uso en trasplante renal en 1999 por la Food and Drug Administration (FDA) ${ }^{4}$ de los Estados Unidos de Norteamérica (USA),es un nuevo agente inmunosupresor del cual existe un número cada vez mayor de reportes que documentan su seguridad en trasplante hepático ortotópico (THO) en adultos ${ }^{4,5}$ y en niños ${ }^{2,3,6,7}$. Su mecanismo de acción y perfil de toxicidad secundaria es diferente de aquel presentado por los IC, ofreciendo potenciales ventajas sobre los esquemas inmunosupresores convencionales ${ }^{2,4-6,8-12}$. Nuestro objetivo es comunicar el caso de una paciente pediátrica sometida a THO, quien presentó rechazo celular agudo (RA) recurrente del injerto y DM secundaria a la inmunosupresión, a quien se le administró SRL como terapia de rescate, con lo que no volvió a presentar RA, se le pudo suspender la insulina y que no presentó complicaciones de la herida quinúrgica secundarias al uso de SRL, luego de cirugía por apendicitis aguda, y, a propósito del caso, revisar el estado actual de la literatura.

Caso clínico. Niña de 10 años de edad, portadora de atresia de vía biliar extrahepática, sometida a operación de Kassai a los 4 meses de vida, que evolucionó con hipertensión portal grave, colangitis a repetición y osteoporosis secundaria. Fue sometida a THO en nuestra unidad, con injerto reducido compatible de cadáver. La inmunosupresión fue en base a esteroides, micofenolato mofetil (Cellcept ${ }^{\circledR}$, Roche Pharmaceuticals, USA) y ciclosporina A (Neoral ${ }^{\circledR}$, Novartis Pharma AG, Suiza). En el postoperatorio inmediato presentó un RA que resolvió con bolos de metilprednisolona (MPS), (10 mg/kg/día por 3 días). A los 3 meses post THO presentó segundo RA que también resolvió con MPS. A los 5 meses, por empeoramiento de bioquímica hepática y sospecha de RA se cambió ciclosporina A (CyA) por Tacrolimus (Prograf $^{\circledR}$, Fujisawa, USA) con normalización de enzimas hepáticas. A los 8 meses con evidente Cushing clínico (prednisona $0,5 \mathrm{mg} / \mathrm{kg} /$ día), apareció una DM insulino dependiente. A los 10 meses se suspendió micofelonato mofetil (MMF), pero un mes después presentó tercer RA que resolvió con MPS, reiniciándose MMF. Cuarto RA a los 15 meses que resolvió con MPS. Entre los 21 y 26 meses presentó diamea por rotavirus, sinusitis aguda, candidiasis oral y lumbago con osteopenia severa que la inmovilizó por dolor y obligó a usar corsé toracoabdominal. Al mes 26 post THO se inició Sirolimus (rapamicina, Rapamune ${ }^{\circledR}$, Wyeth, USA) $1 \mathrm{mg} /$ día, suspendiéndose MMF y bajando progresivamente las dosis de esteroides $(1 \mathrm{mg} / \mathrm{kg} /$ día $)$ y de FK (8 $\mathrm{mg} /$ día, nivel sanguíneo $14,6 \mathrm{ng} / \mathrm{ml}$ ). Al mes 29, estando con dosis de FK de $4 \mathrm{mg} /$ día (nivel sanguíneo 10,9 ng/ml), de esteroides de $0,13 \mathrm{mg} /$ $\mathrm{kg} /$ día y de SRL de $1 \mathrm{mg} /$ día (nivel sanguíneo 5,3 $\mathrm{ng} / \mathrm{ml}$ ) fue exitosamente operada de apendicitis aguda, sin ningún problema de herida operatoria y se logró suspender la insulina por glicemias normales. Al mes 33 mantenía pruebas hepáticas normales en sangre, dosis de esteroides de 0,07 mg/ $\mathrm{kg} /$ día , niveles de FK de $4,2 \mathrm{ng} / \mathrm{ml}$ y de SRL de $5,1 \mathrm{ng} / \mathrm{ml}$ (dosis de $2 \mathrm{mg} /$ día), refiriendo úlceras en mucosa oral autolimitadas en el tiempo, sin necesidad de tratamiento. Al mes 40 post THO se suspendieron los esteroides, la dosis de FK era $5 \mathrm{mg} /$ día (nivel sanguíneo $8,7 \mathrm{ng} / \mathrm{ml}$ ) y de SRL $3 \mathrm{mg} /$ día (nivel sanguíneo $5,9 \mathrm{ng} / \mathrm{ml}$ ). Sin otras complicaciones asociadas a inmunosupresión, ni antes ni después de usar SRL Su lumbago desapareció aunque requiere corsé para deambular y las pruebas hepáticas siguen normales.

\section{DisCUSIÓN}

SRL es un macrólido con propiedades antimicóticas, antiproliferativas e inmunosupresoras. Es estructuralmente similar a los IC, pero tiene mecanismo de acción y efectos secundarios diferentes. SRL se une a la misma proteína a la que se liga el FK, la FK Binding Protein (FKBP), pero este complejo SRL-FKBP no tiene actividad anticalcineurínica. Los IC bloquean la transcripción del gen de interleuquina 2 (IL2), mientras que el SRL-FKBP inhibe la señal post-receptor de IL2, uniéndose a una proteína llamada mammalian target of rapamycin»(mTOR), que normalmente está involucrada en la regulación del ciclo celular, inhibiendo la progresión de los linfocitos $\mathrm{T}$ desde la fase $\mathrm{G}_{1}$ a $S^{4,5,8,12-14}$. En $\mathrm{THO}$, se introdujo primero como terapia de rescate y posteriormente como inmuno- 
supresión primaria $2,4,5,12$, siendo sus indicaciones el rescate en rechazo crónico ${ }^{4,5,8}$, el rechazo agudo resistente a esteroides ${ }^{5,14}$, el rechazo agudo recurrente $^{4,12}$ y el rechazo agudo secundario al retiro de inmunosupresión en ELPT ${ }^{2,11,12}$; reducción o eliminación de la terapia con IC por nefrotoxici$\operatorname{dad}^{2,4,5,10,12,15,16}$, o neurotoxicidad 10,12,17; como inmunosupresión primaria ${ }^{2-4,10,12,14}$ y en casos anecdóticos de receptores con hepatoblastoma ${ }^{11} 0$ con carcinoma hepatocelular ${ }^{4,10}$.

En nuestra paciente, la indicación fue el rechazo agudo recurrente, que no respondía a terapia triasociada con esteroides, MMF e IC. Con SRL no volvió a presentar RA en 14 meses de observación, a pesar de haber retirado el MMF y bajar los esteroides hasta suspenderlos. SRL no causa como efecto secundario $\mathrm{DM}^{4,8,14}$, nefrototoxicidad $^{10,15,16}$ o hipertensión arterial ${ }^{4}$. Nuestra paciente desarrolló DM luego de cambiar su terapia de CyA por FK, agravada probablemente por los esteroides. Jain y cols, comunicaron en su serie de 1.000 pacientes adultos y niños sometidos a THO con FK como inmunosupresión primaria una incidencia de DM de $14 \%$ a un año ${ }^{1}$ y Markell y cols, describieron un mayor efecto diabetogénico del FK comparado con CyA, en pacientes sometidos a THO. Los esteroides, que también son diabetogénicos, actúan por un mecanismo diferente a los IC, que es dosis dependiente, con bajas dosis de esteroides hay bajas tasas de $\mathrm{DM}^{18}$. En nuestra paciente, la administración de SRL permitió bajar en 3 meses las dosis de FK en $50 \%$ y de esteroides casi $90 \%$, lo que posibilitó la normalización de las glicemias y suspensión de la insulina, lo que mejoró su calidad de vida y sobrevida del injerto y la paciente ${ }^{18}$.

Entre las complicaciones descritas por el uso de SRL se encuentran la dislipidemia (hipercolesterolemia e hipertrigliceridemia) ${ }^{4,5,8,9,11-14}$ leucopenia $4,5,8,9,11,12,14$, trombocitopenia $4,8,9,11,12,14$, anemia $^{4,11,14}$, edema de extremidades ${ }^{5,8,11}$, úlceras de la mucosa oral ${ }^{5,11,12}$, dolor muscular y artralgias $^{5,8,12}$, diarrea ${ }^{5,11,12}$, trombosis de arteria hepática ${ }^{4,5,14}$, complicaciones de herida operatoria (infección, dehiscencia) ${ }^{4,5,14}$, complicaciones pulmonares (neumonitis, bronquiolitis obliterante, neumonía) ${ }^{5,11,12}$, infecciones (citomegalovirus, herpes zoster, pneumocistis carinii, sepsis grave $)^{5,11,12}$, hepatotoxicidad 12,19 , dermatitis ${ }^{5}$, náuseas $^{12}$, ELPT $^{12}$ y dificultades para monitorizar en tiempo real los niveles en sangre, ya que SRL tiene vida media prolongada y demora 3 a 5 días en alcanzar el nivel deseado $5,7,8$. Entre 28 y $50 \%$ de los pacientes deben suspender SRL por toxicidad secundaria ${ }^{11,14}$. En nuestra paciente se presentaron úlceras de la mucosa oral, las cuales se describen con una incidencia entre 14 y 37\%, sin embargo, es posible que se resuelvan con solo bajar la dosis de SRL ${ }^{11,12}$.

Si bien han habido comunicaciones anecdóticas de complicaciones de la herida quirúrgica, no existe evidencia directa que señale que SRL tenga impacto negativo sobre la cicatrización de la herida operatoria 4 . Stuart y cols, estudiaron 513 pacientes sometidos a trasplante renal y reportaron que no hubo un incremento significativo en las complicaciones de la herida operatoria al comparar inmunosupresión primaria en un grupo de pacientes con SRL, MMF y esteroides, versus otros dos con CyA, MMF y esteroides y CyA, azatioprina y esteroides ${ }^{20}$. Watson y cols reportaron que 4 de 15 pacientes (27\%) sometidos a THO desarrollaron dehiscencia de la herida, pero los pacientes en este estudio recibieron dosis relativamente altas de SRL (carga inicial de 2 ó $12 \mathrm{mg} / \mathrm{m}^{2}$, seguido de una dosis diaria de $\left.4 \mathrm{mg} / \mathrm{m}^{2}\right)^{21}$. En cambio, otro estudio que comparó un grupo de 170 pacientes sometidos a THO, que recibieron SRL como inmunosupresión primaria, junto con un IC y esteroides contra un grupo control histórico sin SRL, tuvo una prevalencia de complicaciones de la herida operatoria de 12,4\% versus 13,9\% histórico. En este estudio se usaron dosis más bajas de SRL (6 mg el día 0 y luego 2 mg diarios) ${ }^{14}$. Trotter plantea que tanto dosis como niveles sanguíneos altos de SRL pueden asociarse a mayor incidencia de complicaciones de la herida operatoria ${ }^{4}$. Nuestra paciente llevaba 3 meses con dosis y niveles bajos de SRL al momento de la cirugía por apendicitis aguda, no presentando complicaciones, lo que concuerda con lo descrito previamente. En resumen, se presenta el caso de una paciente pediátrica sometida a THO que evolucionó con RA recurrente y DM secundaria a inmunosupresión, quien fue rescatada con SRL, con lo que no presentó nuevos episodios de RA; permitió el retiro del MMF y esteroides; la disminución de la dosis de FK y la suspensión de la insulina, no presentando complicaciones 
graves. Se confirma que SRL es una nueva y segura alternativa a tener en cuenta como

\section{REFERENCIAS}

1. Jain A, Reyes J, Kashyap R, Rohal S, Abu-Elmagd K, Starzl T et aL. What have we leamed about primary liver transplantation under tacrolimus immunosuppression? Long Term follow-up of the first 1000 patients. Ann Surg 1999; 230: 441-8.

2. SindHI R. Sirolimus in pediatric transplant recipients. Transplant Proc 2003; 35 (3 Suppl): 113S$114 \mathrm{~S}$.

3. Sindhi R, Ganjoo J, McGhee W, Mazariegos G, Reyes J. Preliminary immunosuppression withdrawal strategies with sirolimus in children with liver transplant. Transplant Proc 2002; 34: 1972-3.

4. TROtTeR JF. Sirolimus in liver transplantation. Transplant Proc 2003; 35 (Suppl 3A): 193S-200S.

5. NefF GW, Montalbano M, Tzakis AG. Ten years of sirolimus therapy in orthotopic liver transplant recipients. Transplant Proc 2003; 35 (3 Suppl): 209S-216S.

6. KAHAN BD. The potential role of rapimycin in pediatric transplantion as observed from adult studies. Pediatr Transplant 1999; 3: 175-80.

7. Schubert M, Venkataramanan R, Holt DW, Shaw LM, McGhee W, REyes J et aL. Pharmacokinetics of sirolimus and tacrolimus in pediatric transplant patients. Am J Transplant 2004; 4: 767-73.

8. NefF GW, Montalbano M, Slapak-Green G, Berney T, BejARANo P, Joshi A ET AL. A retrospective review of sirolimus (Rapamune) therapy in orthotopic liver transplant recipients diagnostised with chronic rejection. Liver Transplantation 2003; 9: 477-83.

9. Kelly PA, Gruber SA, Behbod F, Kahan BD. Sirolimus, a new, potent immunossuppressive agent. Pharmacotherapy 1997; 17: 1148-56.

10. Chang GJ, Mahanty HD, Quan D, Freise CE, Ascher NL, ROBERTS JP ET AL. Experience with the use of sirolimus in liver transplantation-use in patients for whom calcineurin inhibitors are contraindicated. Liver Transpl 2000; 6: 734-40.

11. JimÉnEZ-Rivera C, Avitzur Y, FeCteau AH, Jones N, GRANT D, NG VL. Sirolimus for pediatric liver transplant recipients with post-transplant lymphoproliferative disease and hepatoblastoma. Pediatr Transplant 2004; 8: 243-8. terapia inmunosupresora de rescate en pacientes sometidos a THO.

12. Sindhi R, Webber S, Venkataramanan R, McGhee W, Рнiшps S, Sмith A eт al. Sirolimus for rescue and primary immunosuppression in transplanted children receiving tacrolimus. Transplantation 2001; 72: 851-4.

13. Parrot NR. Immunosuppression: «What's new?» En Transplantation Surgery: Current Dilemmas. Edited by John L R Forsythe. W.B. Saunders Company Limited. Second edition 2001; 101131.

14. Dunkelberg JC, Troter JF, Wachs M, Kugelmas M, Streinberg T, Everson GT et al. Sirolimus as primary immunosuppression in liver transplantation is not associated with hepatic artery or wound complications. Liver Transpl 2003; 9: 463-8.

15. Fairbanks KD, Eustace JA, Fine D, Thuluvath PJ. Renal function improves in liver transplant recipients when switched from a calcineurin inhibitor to sirolimus. Liver Transpl 2003; 9: 1079-85.

16. Neff GW, Montalbano M, Slapk-Green G, Meyer D, Berney T, SAFDar K et al. Sirolimus therapy in orthotopic liver transplant recipients with calcineurin inhibitor related chronic renal insufficiency. Transpl Prc 2003; 35: 3029-31.

17. Maramattom BV, Eelco FMW. Sirolimus may not cause neurotoxicity in kidney and liver transplant recipients. Neurology 2004; 63: 1958-9.

18. MARKELL M. New-onset diabetes mellitus in transplant patients: pathogenesis, complications, and management. Am J Kidney Dis 2004; 43: 953-65.

19. NefF GW, Ruiz P, Madariaga JR, Nishida S, MontalBANO M, MEYer D ET AL. Sirolimus associated hepatotoxicity in liver transplantation. Ann Pharmacother 2004; 38: 1593-6.

20. Fiechner SM, Zhou L, Derweesh I, Mastroianni B, SAVAS K, GoldFarb D et al. The impact of sirolimus, mycophenolate mofetil, cyclosporine, azathioprne, and steroids on wound healing in 513 kidneytransplant recipients. Transplantation 2003; 76 : 1729-34.

21. Watson CJ, Friend PJ, Jamieson NV, Frick TW, AleXANDER G, Gimson AE et al. Sirolimus: a potent new immunosuppressant for liver transplantation. Transplantation 1999; 67: 505-9. 